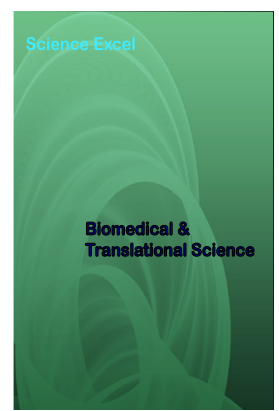

Correspondence

Dr. George Pak-Heng Leung,

2/F, 21 Sassoon Road, Li Ka Shing Faculty of Medicine, Laboratory Block, Faculty of Medicine Building, Department of Pharmacology and Pharmacy, University of Hong Kong, Hong Kong SAR, China. Tel: +852 39176861

E-mail: gphleung@hku.hk

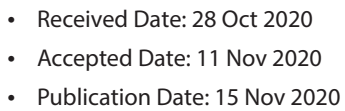

Copyright

(c) 2020 Science Excel. This is an openaccess article distributed under the terms of the Creative Commons Attribution 4.0 International license.

\title{
Comparison of the major chemical constituents and antioxidant effects in Amauroderma rugosum and Ganoderma lucidum
}

\author{
Renkai Li ${ }^{1}$, Jingjing Li ${ }^{1}$, Timothy Man-Yau Cheung ${ }^{2}$, Bryan Siu-Yin $\mathrm{Ho}^{2}$, George \\ Pak-Heng Leung ${ }^{1, *}$
}

'Department of Pharmacology and Pharmacy, The University of Hong Kong, Hong Kong, China; ${ }^{2}$ Tian Ran Healthcare Limited, Hong Kong

\begin{abstract}
Aging is a major risk factor for many diseases, including cardiovascular diseases, neurological disorders, cancer and diabetes mellitus. Oxidative stress plays a key role in the aging process. Amauroderma rugosum is an edible mushroom that has rarely been studied. The aims of this study were to compare the major chemical constituents and to investigate the antioxidant effects of Amauroderma lucidum and Ganoderma lucidum. The water extract of Amauroderma lucidum contained a higher amount of total phenolic compounds than that of Ganoderma lucidum. The total polysaccharide and triterpene content in water extracts of Amauroderma rugosum and Ganoderma lucidum did not significantly differ. The water extract of Amauroderma rugosum demonstrated free radical scavenging capacity and could reduce doxorubicin-induced damage in H9c2 cardiomyoblasts. Amauroderma rugosum has stronger antioxidant and cellular protective effects than Ganoderma lucidum. Amauroderma rugosum may be beneficial in healthy aging, and further study should be encouraged.
\end{abstract}

\section{Introduction}

Aging is a progressive loss of tissue and organ functions over time. Although various hypotheses have been proposed to explain the cellular and molecular mechanisms of aging, many studies have clarified that ageassociated functional loss is essentially due to the accumulation of oxidative stressinduced molecular damage [1].

The generation of free radicals and related reactive oxygen species is an inevitable consequence of aerobic life and serves many useful purposes; for instance, infectious disease might occur without them. However, when the oxidant/antioxidant balance is disrupted and tilts toward an oxidative status (a condition known as oxidative stress), harmful effects to cell survival may occur, including lipid peroxidation and oxidative modification of proteins and nucleic acids. Indeed, oxidative stress has been implicated in various pathologies including cardiovascular and neurodegenerative diseases, cancers, diabetes mellitus and cataracts, most of which are age-related.

The development of antioxidant therapeutic agents has received considerable interest, with the aim of treating or slowing the onset of aging-related diseases. Healthcare products containing edible and medicinal mushrooms such as Ganoderma Lucidum (also known as Lingzhi in Chinese) are very popular because they have been suggested to be beneficial in boosting the immune system. Studies on the antioxidant and antiaging effects of Ganoderma Lucidum are emerging [2]. Although the beneficial effects of Ganoderma Lucidum have been widely studied, other similar species of mushroom might potentially be better than Ganoderma Lucidum or might have certain beneficial properties that Ganoderma Lucidum lacks.

Amauroderma rugosum is a dietary mushroom in the Ganodermataceae family. This mushroom has a black stipe and a white surface covered with numerous pores. A notable characteristic is that the mushroom's surface becomes red when it is scratched. Hence, it is also known as "blood Lingzhi" in Chinese. Although Amauroderma rugosum is commonly consumed by people in China and South Asia, few scientific studies have explored its medicinal or nutritional value. In this study, we sought to compare the major chemical constituents and antioxidant effects of Amauroderma rugosum and Ganoderma Lucidum. 


\section{Materials and methods}

Chemicals and reagents

Dulbecco's modified Eagle's medium (DMEM), fetal bovine serum (FBS), penicillin-streptomycin and $0.25 \%$ (w/v) trypsin containing $1 \mathrm{mM}$ ethylenediaminetetraacetic acid were purchased from Invitrogen (Carlsbad, CA), and 6-OHDA, 2,2-diphenyl-1-picryl-hydrazyl-hydrate (DPPH), dimethyl sulfoxide and 3-(4,5-dimethylthiazol-2-yl)-2,5diphenyltetrazoliumbromide (MTT) were purchased from Sigma Aldrich (St. Louis, MO). All chemicals were dissolved in appropriate solvents and stored at $-20^{\circ} \mathrm{C}$ before use to maintain their chemical stability.

\section{Reflux extraction}

Fruiting bodies of Amauroderma rugosum and Ganoderma Lucidum (Figure 1) were provided by Hong Kong Ganoderma Centre Limited (Hong Kong, China), an organic farm that had been granted an organic crop production certificate by the Hong Kong Organic Resource Centre. The samples were dried in an oven and ground into powder. A reflux system for the extraction process was used to prepare the crude extract. Two grams of powdered sample was extracted with $50 \mathrm{~mL}$ distilled water at $95 \pm 2^{\circ} \mathrm{C}$ for $60 \mathrm{~min}$. The crude extract was centrifuged at $4000 \mathrm{rpm}$ for $20 \mathrm{~min}$. Afterward, the supernatant was collected, and the sample residue was re-extracted twice via the steps described above. Subsequently, all extracts were pooled, filtered and concentrated to $80 \mathrm{~mL}$ with a rotary evaporator. The extract was stored at $-20^{\circ} \mathrm{C}$ until further use.

Determination of total phenolic compounds, polysaccharides and triterpenes

To measure the total phenolic content of the extracts, we added $50 \mu \mathrm{L}$ of $10 \%$ Folin-Ciocalteu phenol reagent to 50 $\mu \mathrm{L}$ extract and incubated the mixture in the dark at room temperature for $3 \mathrm{~min}$. Afterward, $100 \mu \mathrm{L}$ of $10 \% \mathrm{Na}_{2} \mathrm{CO}_{3}$ was added to the mixture for $1 \mathrm{~h}$. The absorbance at $750 \mathrm{~nm}$ was measured with a microplate absorbance reader. Gallic acid was used as a standard phenolic compound. All determinations were expressed as mg gallic acid equivalent per $\mathrm{g}$ (mg GAE/g).

Before measurement of total polysaccharides, $0.1 \mathrm{~mL}$ extract was precipitated with $1 \mathrm{~mL}$ of $95 \%$ ethanol overnight at $4^{\circ} \mathrm{C}$. The precipitate was collected by centrifugation at 10,000 $\mathrm{rpm}$ for $10 \mathrm{~min}$ at $4^{\circ} \mathrm{C}$. Then, the precipitate was dissolved in $50 \mu \mathrm{L}$ water. The total polysaccharide content was measured by addition of $2.5 \mu \mathrm{L}$ phenol (80\%) and then $125 \mu \mathrm{L}$ concentrated sulfuric acid. After incubation for $10 \mathrm{~min}$, the mixture was shaken and incubated at $30^{\circ} \mathrm{C}$ for $20 \mathrm{~min}$. The absorbance at $490 \mathrm{~nm}$ was measured with a microplate absorbance reader with glucose as a standard. The results were expressed as mg glucose equivalent per $\mathrm{g}$ (mg GE/g).

To measure the total triterpenes, we transferred $100 \mu \mathrm{L}$ extract to a $15 \mathrm{~mL}$ tube and evaporated it to dryness using

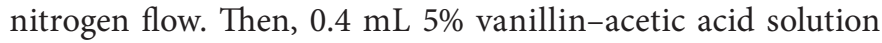
and $1 \mathrm{~mL}$ perchloric acid were added into the tube, mixed and incubated at $60^{\circ} \mathrm{C}$ for $15 \mathrm{~min}$. Afterward, $5 \mathrm{~mL}$ acetic acid was added and incubated at room temperature for $15 \mathrm{~min}$. The absorbance at $549 \mathrm{~nm}$ was measured with a microplate absorbance reader. A solution of oleanolic acid was used as the standard. The results were expressed as mg oleanolic acid equivalent per $\mathrm{g}(\mathrm{mg} \mathrm{OAE} / \mathrm{g})$

\section{$\mathrm{DPPH}$ assay}

The free radical scavenging capacity of the extract was measured with DPPH assays. Briefly, $5 \mu \mathrm{L}$ extract $(40 \mathrm{mg} /$ $\mathrm{mL})$ was mixed with $195 \mu \mathrm{L}$ of DPPH solution $(24 \mathrm{mg} / \mathrm{L})$ in a 96-well plate. The reaction proceeded in the dark for $60 \mathrm{~min}$. Afterward, the absorbance of the reaction mixture at $515 \mathrm{~nm}$ was measured with a microplate absorbance reader.
A

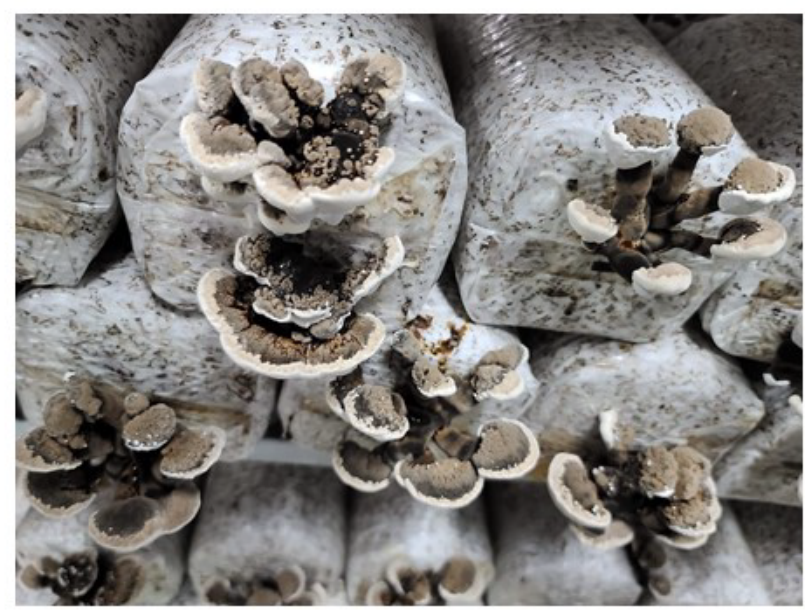

B

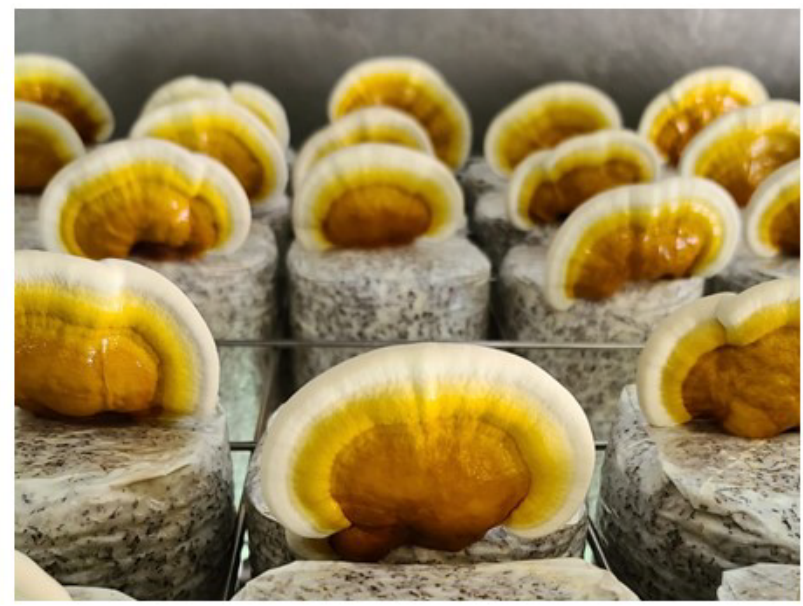

Figure 1. The typical appearance of (A) Amauroderma Rugosum and (B) Ganoderma lucidum. 
A

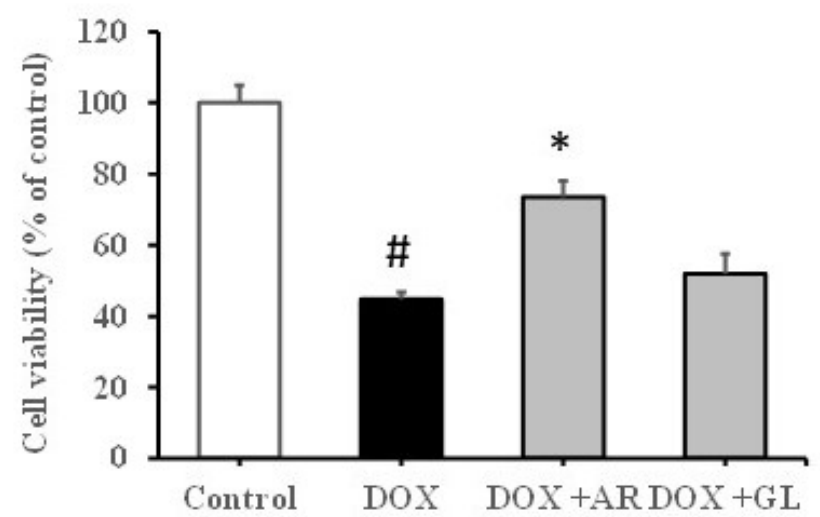

B

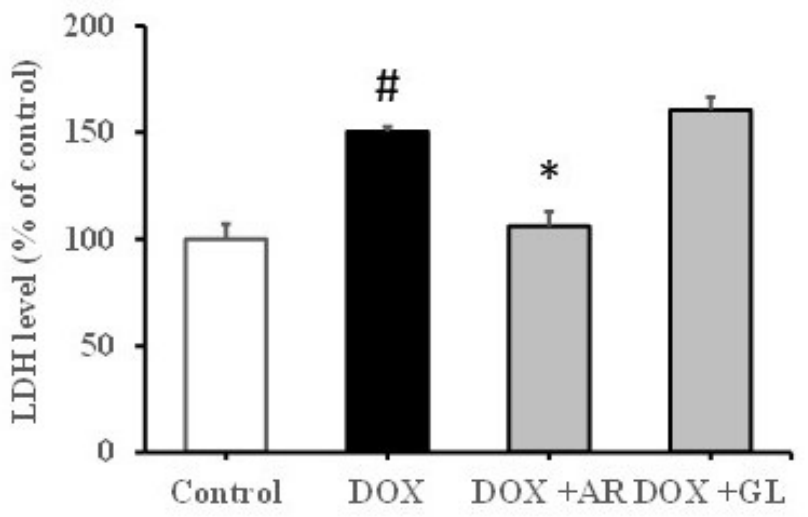

Figure 2. Protective effects of water extract of Amauroderma rugosum and Ganoderma lucidum on H9C2 rat cardiomyoblasts under oxidative stress. H9C2 cells were left untreated (control) or were treated with doxorubicin (Dox; $0.5 \mu \mathrm{M}$ ) in the absence or presence of $2 \mathrm{mg} / \mathrm{mL}$ water extract of Amauroderma rugosum (AR) or Ganoderma lucidum (GL) for $24 \mathrm{~h}$. (A) Cell viability and (B) LDH levels were determined with MTT and biochemical assays, respectively. Values are means \pm S.D. $(n=3)$. $\# p<0.05$ versus control; $\# p<0.05$ versus control; $* p<0.05$ versus Dox.

Table 1. Total content of phenolic compounds, polysaccharides and triterpenes in water extracts of Amauroderma rugosum and Ganoderma lucidum. Values are means \pm S.D. $(\mathrm{n}=5) .{ }^{*} \mathrm{p}<0.05$ compared with Ganoderma lucidum.

\begin{tabular}{|l|c|c|c|}
\hline & \multicolumn{3}{|c|}{ Total Contents (mg/g) } \\
\hline & Phenolic compounds & Polysaccharides & Triterpenes \\
\hline Amauroderma rugosum & $5.529 \pm 0.111^{*}$ & $1.119 \pm 0.230$ & $3.196 \pm 0.136$ \\
\hline Ganoderma lucidum & $3.995 \pm 0.076$ & $1.352 \pm 0.193$ & $3.423 \pm 0.185$ \\
\hline
\end{tabular}

\section{Cell culture and treatment}

$\mathrm{H} 9 \mathrm{c} 2$ rat cardiomyoblasts were obtained from the American Type Culture Collection (Manassas, VA). The cells were cultured in DMEM supplemented with $10 \%$ heatinactivated FBS and 1\% penicillin-streptomycin, and then incubated at $37^{\circ} \mathrm{C}$ in a humidified atmosphere with $5 \% \mathrm{CO}_{2}$. For the cell viability and LDH assays, H9c2 cells in DMEM with low serum $(0.5 \%$ FBS) were seeded in 96-well plates. The cells were incubated with Amauroderma rugosum and Ganoderma Lucidum extracts $(40 \mathrm{mg} / \mathrm{mL})$ for $2 \mathrm{~h}$, and then treated with $0.5 \mu \mathrm{M}$ doxorubicin for $24 \mathrm{~h}$.

Cell viability assay

Cell viability was measured with MTT assays according to the manufacturer's protocol. In brief, the culture medium was discarded, and the cells were incubated with MTT solution (at a final concentration of $0.5 \mathrm{mg} / \mathrm{mL}$ ) for $4 \mathrm{~h}$ at $37^{\circ} \mathrm{C}$. Dimethyl sulfoxide was then added to lyse the cells and dissolve the violet formazan crystals that had formed inside the cells. The absorbance at $570 \mathrm{~nm}$ was measured with a microplate absorbance reader.

\section{LDH assay}

Cellular injury was determined by measurement of the $\mathrm{LDH}$ released into the culture medium. $\mathrm{LDH}$ activity was measured with a detection kit according to the manufacturer's instructions. The absorbance at $490 \mathrm{~nm}$ was measured with a microplate absorbance reader.

\section{Data and statistical analysis}

Data are expressed as the mean \pm standard deviation (SD) of at least three independent experiments. Statistical analyses were performed with one-way ANOVA followed by Tukey's multiple comparison test (for two or more groups) in GraphPad Prism 6.0 software (GraphPad Software Inc., San Diego, CA, USA). $\mathrm{p}<0.05$ was considered statistically significant. 


\section{Results}

Chemical content of Amauroderma rugosum and Ganoderma Lucidum extract

The major chemical composition of Amauroderma rugosum and Ganoderma Lucidum extract, including total phenolic compounds, polysaccharides and triterpenes, was measured with chemical assays (Table 1). The content of total phenolic compounds of water extracts of Amauroderma rugosum was $5.529 \pm 0.110 \mathrm{mg} \mathrm{GAE} / \mathrm{g}$ dry weight. This value was $38.4 \%$ higher than that of Ganoderma Lucidum. The total polysaccharide content of water extract of Amauroderma rugosum was $1.119 \pm 0.230 \mathrm{mg}$ GE/g dry weight, which was not statistically significant different from that of Ganoderma Lucidum. The total triterpene content of water extract of Amauroderma rugosum was $3.196 \pm 0.136 \mathrm{mg}$ OAE/g dry weight, which was also not statistically significantly different from that of Ganoderma Lucidum.

Antioxidant capacity of Amauroderma rugosum and Ganoderma Lucidum extracts in DPPH assays

The antioxidant capacity of water extracts of Amauroderma rugosum and Ganoderma Lucidum, was studied with DPPH assays. The scavenging ability of Amauroderma rugosum extract and Ganoderma Lucidum extract was $59.2 \pm 1.8$ and $39.4 \pm 1.5 \%$, respectively.

Protective effects of Amauroderma rugosum and Ganoderma Lucidum extracts on $\mathrm{H} 9 \mathrm{c} 2$ cardiomyoblasts

H9c2 cells were used as a model to compare the cellular protective effects of Amauroderma rugosum and Ganoderma Lucidum extracts. The MTT assays showed that the viability of H9c2 cells decreased by $55.2 \%$ in the presence of doxorubicin but by only $26.4 \%$ when the cells were pre-incubated with Amauroderma rugosum extract (Figure 2A). LDH was used as a marker of cellular damage. The LDH level increased by $50.5 \%$ under doxorubicin treatment, whereas this increase was abolished by Amauroderma rugosum extract (Figure 2B). Ganoderma Lucidum extract had no effect on both the cell viability and $\mathrm{LDH}$ level in $\mathrm{H} 9 \mathrm{c} 2$ cells. .

\section{Discussion}

Ganoderma Lucidum is the best-known and most popular edible and medicinal mushroom in Asia. In traditional Chinese medicine, it is used to promote health and longevity [3]. Numerous studies have demonstrated that Ganoderma Lucidum exerts significant beneficial effects in neurodegeneration, diabetes mellitus, cardiovascular diseases and tumor development [4]. These promising pharmacological effects of Ganoderma Lucidum are at least partly attributed to its antioxidant and free radical scavenging activity. For instance, Ganoderma Lucidum extract has been found to ameliorate MPTP-induced Parkinsonism and protect dopaminergic neurons against oxidative stress via regulating mitochondrial function, autophagy and apoptosis [5]. In a rat model, pre-administration of Ganoderma Lucidum has been found to prevent mitochondrial dysfunction and apoptosis of hippocampal neurons by alleviating oxidative stress [6]. Other dietary mushrooms in the Ganodermataceae family might also potentially exhibit beneficial effects for the prevention or treatment of oxidative stress-related diseases. Amauroderma rugosum is a species in the Ganodermataceae family whose chemical constituents and pharmacological effects have rarely been explored. Only two studies have described the chemical constituents of Amauroderma rugosum; these studies have reported that ethanolic extract of Amauroderma rugosum contains phenolic compounds $[7,8]$. However, no information was reported about polysaccharides and triterpenes, which are well-known active ingredients in Ganoderma Lucidum. Moreover, the major chemical contents of aqueous extract of Amauroderma rugosum had not been explored. This information is important because water decoction, but not ethanol extraction, is the traditional and most common method of consumption of those mushrooms.

In addition to chemical analysis, pharmacological studies associated with Amauroderma rugosum are also very rare. Only one publication has reported lipid modulating effects [9], one has reported antioxidant effects [10], one has reported anti-inflammatory effects [8], one has reported morphological assessment [11], one has reported the identification of lignin peroxidase [12], and one has reported nutritional composition [7]. In addition, one report described an acute toxicological study [13] demonstrating that Amauroderma rugosum is biologically safe. In that study, oral administration of a dose of Amauroderma rugosum powder (2000 mg/kg) had no adverse effects on the growth rate or hematological and clinical biochemical parameters in an animal model. Histological studies also showed that the treatments did not induce any pathological changes in the organs.

In the present study, the results of chemical assays demonstrated that aqueous extract of Amauroderma rugosum contains phenolic compounds, polysaccharides and triterpenes. The content of total polysaccharides and triterpenes of Amauroderma rugosum extract was comparable to that of Ganoderma Lucidum extract. Interestingly, the content of total phenolic compounds of Amauroderma rugosum extract was unexpectedly much higher than that of Ganoderma Lucidum extract. Indeed, numerous studies have shown that phenolic content is highly correlated with antioxidant activity [14]. Therefore, we sought to investigate whether Amauroderma rugosum extract might potentially have antioxidant activity. Ethyl acetate extract of Amauroderma rugosum mycelium has been reported to have antioxidant and anti-inflammatory effects in lipopolysaccharide-stimulated RAW 264.7 cells [7]. Our results in this study demonstrated that the aqueous extract of Amauroderma rugosum also exerted significant antioxidative activity in DPPH assays. In agreement with the higher phenolic content of Amauroderma rugosum, the antioxidant capacity of Amauroderma rugosum extract was stronger than that of Ganoderma Lucidum extract. Moreover, the antioxidant effect of Amauroderma rugosum extract was further examined in an in vitro cell model involving doxorubicin. Although doxorubicin is a highly effective anticancer drug, its clinical use is limited by its cardiotoxicity, which is due to doxorubicin-induced oxidative stress. Doxorubicin induces death of $\mathrm{H} 9 \mathrm{c} 2$ rat cardiomyoblasts. However, this detrimental effect of doxorubicin decreased when the cells were treated with Amauroderma rugosum extract. The protective effect of Amauroderma rugosum was also stronger than that of Ganoderma Lucidum.

Oxidative stress is widely believed to be involved in the pathogenesis of many age-related diseases, such as 
neurodegenerative diseases, cardiovascular diseases and cancer. Some antioxidants have been demonstrated to be effective in the prevention or treatment of oxidative stressrelated diseases [15]. The most extensively studied antioxidants are vitamin $A$ and its precursor $\beta$-carotene, vitamin $C$, and vitamin E. Several large observational studies have been conducted on the effects of the intake of different vitamins on the risk of cardiovascular diseases [15]. However, systematic reviews and meta-analyses conducted by Cochrane group investigators studying the effects of vitamins on all-cause mortality have indicated many conflicting results. In some trials, vitamins did not appear to significantly affect mortality, but in several other trials, they were administered alone or in combination and were associated with a significant increase in all-cause mortality [16]. Although the reason for these disappointing results is unclear, these findings led to the conclusion that vitamins cannot be used as effective antioxidant therapeutic agents.

$\mathrm{N}$-acetylcysteine is a precursor of glutathione. Although $\mathrm{N}$-acetylcysteine is considered a safe substance, the results of clinical trials have sometimes been controversial or incomplete [17]. Coenzyme $\mathrm{Q}_{10}\left(\mathrm{CoQ}_{10}\right)$ is an endogenous lipid that participates in mitochondrial respiratory chain reactions. Numerous pathological processes are associated with primary and secondary $\mathrm{CoQ}_{10}$ deficits, including mitochondrial diseases, fibromyalgia, cardiovascular diseases, neurological disorders, cancer and diabetes mellitus. Although $\mathrm{CoQ}_{10}$ levels decreased with aging in humans, this effect is not seen in all species or all tissues [18]. In addition, whether lower $\mathrm{CoQ}_{10}$ levels have a part in aging or are merely an inconsequential cellular response to aging is unknown. Despite the current public interest in supplementation with $\mathrm{CoQ}_{10}$, current evidence is insufficient to recommend $\mathrm{CoQ}_{10}$ supplementation as an anti-aging antioxidant therapy [18]. The effects of $\mathrm{CoQ}_{10}$ on specific cardiovascular risk factors, such as blood pressure, dyslipidemia, and glycemic control, are less impressive. Therefore, current evidence does not support the routine use of $\mathrm{CoQ}_{10}$ in patients with coronary heart disease.

Our findings in this study have significant implications. Amauroderma rugosum may be beneficial in healthy aging because of its excellent antioxidant and cellular protecting effects. Although the active ingredients in Amauroderma rugosum have not yet be defined, phenolic compounds are most likely to be involved. Polyphenols are secondary metabolites of plants, and are widely found in fruits, vegetables, cereals and beverages. An epidemiological study on French people has indicated a low incidence of cardiovascular diseases in this population despite high dietary consumption of saturated fat [19]. This paradox might be attributed to high wine consumption in this population, which provides high amounts of polyphenols, particularly resveratrol, which decreases platelet aggregation [20]. Wine and/or resveratrol can also decrease the incidence of other pathologies, such as neurodegenerative conditions, cancer and osteoporosis. Nevertheless, polyphenols are characterized by their very low bioavailability: $<1 \%$ of the ingested amount reaches the plasma [21]. Large amounts of wine/polyphenols must be consumed to produce significant antioxidant effects. Therefore, the pharmacokinetic and pharmacodynamic properties of bioactive ingredients in Amauroderma rugosum warrant further study. Development of Amauroderma rugosum as a functional food product is promising, because Amauroderma rugosum grows quickly under cultivation conditions, and thus the production cost is relatively low.

In conclusion, our results provide the first evidence that the total phenolic compounds and antioxidant capacity of Amauroderma rugosum extract are higher than those of Ganoderma Lucidum. We propose that Amauroderma rugosum extract may potentially decrease the occurrence or slow the progress of oxidative stress-associated disorders.

\section{Conflict of Interest}

The authors declare no conflict of interest.

\section{References}

1. Harman D. Aging: a theory based on free radical and radiation chemistry. J Gerontol. 1956;11(3):298-300.

2. Wang J, Cao B, Zhao H, Feng J. Emerging roles of Ganoderma Lucidum in anti-aging. Aging Dis. 2017;8(6):691-707.

3. Sliva D. Ganoderma lucidum (Reishi) in cancer treatment. Integr Cancer Ther. 2013; 2(4):358-364.

4. Lin Z, Deng A. Antioxidative and free radical scavenging activity of Ganoderma (Lingzhi). Adv Exp Med Biol. 2019;1182:271-297.

5. Ren ZL, Wang CD, Wang T, et al. Ganoderma lucidum extract ameliorates MPTP-induced parkinsonism and protects dopaminergic neurons from oxidative stress via regulating mitochondrial function, autophagy, and apoptosis. Acta Pharmacol Sin. 2019;40(4):441-450.

6. Zhou Y, Qu ZQ, Zeng YS, et al. Neuroprotective effect of preadministration with Ganoderma lucidum spore on rat hippocampus. Exp Toxicol Pathol. 2012;64(7-8):673-680.

7. Chan PM, Kanagasabapathy G, Tan YS, Sabaratnam V, Kuppusamy UR. Amauroderma rugosum (Blume \& T. Nees) Torrend: Nutritional composition and antioxidant and potential anti-inflammatory properties. Evid Based Complement Alternat Med. 2013:304713.

8. Chan PM, Tan YS, Chua KH, Sabaratnam V, Kuppusamy UR. Attenuation of inflammatory mediators (TNF-alpha and nitric oxide) and up-regulation of IL-10 by wild and domesticated basidiocarps of Amauroderma rugosum (Blume \& T. Nees) Torrend in LPS-Stimulated RAW264.7 Cells. Plos One. 2015:10(10): e0139593.

9. Seng CK, Abdullah N, Aminudin N. Antioxidative and inhibitory effects of the fruiting body of black lingzhi mushroom, Amauroderma rugosum (Agaricomycetes), on LDL oxidation and HMG-CoA reductase activity. Int J Med Mushrooms. 2017b;19(9):797-807.

10. Seng CK, Abdullah N, Aminudin N. Lipid-modulating effect of black lingzhi medicinal mushroom, Amauroderma rugosum (Agaricomycetes), on oleate-induced human hepatocellular liver carcinoma cells. Int J Med Mushrooms. 2017a;19(12):1101-1111.

11. Costa-Rezende DH, Robledo GL, Góes-Neto A, Reck MA, Crespo E, Drechsler-Santos ER. Morphological reassessment and molecular phylogenetic analyses of Amauroderma s.lat. raised new perspectives in the generic classification of the Ganodermataceae family. Persoonia. 2017;39:254-269.

12. Jong WYL, Show PL, Ling TC, Tan YS. Recovery of lignin peroxidase from submerged liquid fermentation of Amauroderma rugosum (Blume \& T. Nees) Torrend using polyethylene glycol/salt aqueous two-phase system. J Biosci Bioeng. 2017;124(1):91-98.

13. Fung SY, Tan NH, Kong BH, Lee SS, Tan YS, Sabaratnam V. 
Acute toxicity study and the in vitro cytotoxicity of a black lingzhi medicinal mushroom, Amauroderma rugosum (Agaricomycetes), from Malaysia. Int J Med Mushrooms. 2017;19(12):1093-1099.

14. Kalaycioglu Z, Erim FB. Total phenolic contents, antioxidant activities, and bioactive ingredients of juices from pomegranate cultivars worldwide. Food Chem. 2017;221:496-507.

15. Firuzi O, Miri R, Tavakkoli M, Saso L. Antioxidant therapy: current status and future prospects. Curr Med Chem. 2011;18(25):3871-3888

16. Bjelakovic G, Nikolova D, Gluud LL, Simonetti RG, Gluud C. Mortality in randomized trials of antioxidant supplements for primary and secondary prevention: systematic review and meta-analysis. JAMA. 2007;297(8):842-887.
17. Šalamon S, Kramar B, Marolt TP, Poljšak B, Milisav I. Medical and dietary uses of $\mathrm{N}$-acetylcysteine. Antioxidants (Basel). 2019;8(5):111.

18. Barcelos IP, Haas RH. CoQ10 and Aging. Biology (Basel). 2019; 8(2): 28.

19. Artero A, Artero A, Tarín JJ, Cano A. The impact of moderate wine consumption on health. Maturitas. 2015;80(1):3-13.

20. Renaud S, de Lorgeril M. Wine, alcohol, platelets, and the French paradox for coronary heart disease. Lancet. 1992;339(8808):1523-1526.

21. McDougall GJ, Dobson P, Smith P, Blake A, Stewart D. Assessing potential bioavailability of raspberry anthocyanins using an in vitro digestion system. J Agric Food Chem. 2005;53(15):58965904. 